\title{
Synchrotron Based X-ray Microscopy and Micro-Spectroscopy: Multidisciplinary Tools
}

\author{
J. Susini
}

European Synchrotron Radiation Facility, BP220, F-38043 Grenoble Cedex, France

The unique properties of X-rays make them a powerful probing radiation for use in microscopy techniques. For example, soft X-ray microscopes have established capabilities in absorption contrast imaging of thick hydrated biological material in near-native environments at spatial resolutions well beyond those achievable with visible light microscopy. In the past decade there has been a strong tendency in X-ray microscopy to develop alternative contrast mechanisms and spectroscopic methods, which can provide both valuable complementary information on the sample nature and/or reduce the necessary radiation doses. Simultaneously, the development of high brilliance high energy X-ray sources coupled with advances in manufacturing technologies of focusing optics has led to significant improvements in sub-micrometer probes for spectroscopy, diffraction and imaging applications in the multi-keV 'hard' X-ray range. Both by extrapolation of the experience gained in the soft X-ray regime and by the development of new techniques, 'hard' Xray microscopes now offer a complementary analytical tool which can contribute to a wide range of existing and new applications of X-ray microscopy.

Among the 40 beamlines in operation at the European Synchrotron Radiation Facility (Grenoble, France), three beamlines are fully dedicated to X-ray microscopy and micro-spectroscopy techniques in the multi-keV energy range. The main fields of applications are driven by the unique attributes of $\mathrm{X}$-ray microscopy in this spectral range: i) access to K-absorption edges and fluorescence emission lines of medium-light elements and L,M - edges of heavy materials for micro-spectroscopy, chemical or trace element mapping; ii) higher penetration depths compared to soft X-rays allowing imaging of thicker samples; iii) favorable wavelengths for diffraction studies and iv) generally large focal lengths and depths of focus which are advantageous for the use of specific sample environments (in-situ, high pressure, controlled temperatures...). Typical experiments can be broadly divided into two categories: i) morphological studies which require high spatial resolution and are therefore well adapted to 2D or 3D transmission full-field microscopy. ii) studies dealing with co-localization and/or speciation of trace elements in heterogeneous matrices at the micron scale. Scanning X-ray microscopy, in transmission and/or fluorescence modes, tends to be better suited for the latter cases, which often require both low detection limits and spectroscopic analysis capabilities. An increasing number of experiments require a complete analysis of the same sample by screening light and heavy elements.

The presentation will focus on the ID21 instrument which is a beamline dedicated to multi-KeV Xray imaging and spectro-microscopy [1]. The beamline is installed on a low-beta straight section, which is equipped with three different undulators and provides beam for two independent endstations on two separate branch-lines: The Scanning X-ray Microscope (SXM) served by the "direct" branch-line, is equipped with a fixed-exit silicon double crystal monochromator operating in the $2-8 \mathrm{keV}$ energy range, and the full-field imaging transmission X-ray microscope (TXM), is served by the side-branch and optimized for imaging techniques at $4 \mathrm{keV}$. Both microscopes use zone-plates as focusing lenses. The beamline covers several disciplines over a broad energy range. 
Therefore various types of zone plates are currently needed for each kind of application. i) Fluorescence or diffraction applications in the multi-keV energy range require large diameter and medium-resolution (thus long focal length) zone-plates, offering high flux and a better access to the sample. ii) Full-field X-ray microscopy necessitates high-resolution zone-plates, to be used as objective lenses and large diameter zone-plates for condensers. Several collaborations were therefore initiated with different laboratories with particular emphasis upon high efficiency zone-plates for the 2-8 $\mathrm{keV}$ range on one hand $[2,3]$ and high-resolution zone plates for the $\mathrm{Ca} \mathrm{K}$-edge region $(\sim 4 \mathrm{keV})$ on the other [4]. The high spatial resolution focusing combined with spectroscopic methods in the few $\mathrm{keV}$ domain has demonstrated unique capabilities in a number of experiments from diverse research fields such as Biology [5, 6], Geology [7, 8, 9], Environmental Sciences [10] and Materials Science $[11,12]$.

Multi-keV X-ray microscopy often suffers from a lack of absorption resulting in low contrast images. Furthermore, the use of absorption contrast can subject the specimen to high radiation doses leading to possible structural changes. Even for radiation hard materials, many of the samples imaged using fluorescence yield are insufficiently absorbing to provide high contrast images in transmission mode. Therefore accurate morphological localization of trace elements is difficult or even impossible. The development of phase contrast methods fully compatible with detection in fluorescence yield is therefore essential. Those general considerations led us to focus our R\&D program on the investigation of various possible optical schemes for phase contrast imaging. Three original strategies are currently developed on ID21: Differential Phase Contrast (DPC) using configured detectors on the SXM [13]; Differential Interferential Contrast (DIC) with a configured zone-plate on both SXM and TXM $[14,15]$ and finally, Zernike X-ray microscopy on the TXM [16, 17] were successfully developed and are now routinely used.

References

[1] J. Susini et al., Surface Review and Letters, 9(1) (2002) 203.

[2] E. Di Fabrizio et al., Nature, 401 (1999) 89.

[3] C. David et al., Appl. Phys. Letters, 77(23) (2000) 3851.

[4] M. Panitz et al., AIP Conference Proceedings, 507 (2000) 676.

[5] C. Merigoux et al., Biochimica \& Biophysica Acta, 1619 (2003) 53.

[6] T. Ueki et al., Zoological Science, 19(I) (2002) 27.

[7] J.P. Cuif et al., Geochimica \& Cosmochimica Acta, 67(1) (2003) 75.

[8] N. Métrich et al., Geophysical Research Letters, 29(11), (2002) 33.

[9] I. Bihannic et al., Langmuir, 17 (2001) 4144.

[10] J. Prietzel et al., European Journal of Soil Science, 54(3) (2003) 423

[11] P.Bergonzo et al., Diamond and Relat. Mater., 11 (2002) 418.

[12] L. Di Gaspare et al., Solid State Communications, 122 (2002) 359.

[13] G. R. Morrison et al., J. Phys. IV, 104 (2003) 547.

[14] T. Wilhein et al., Appl. Phys. Letters, 78(14) (2001) 2082.

[15] B. Kaulich et al., JOSA A, 19 (4) (2002) 797.

[16] Schneider et al., Appl. Phys. Letters, 78(13) (2001) 1936.

[17] U. Neuhaeusler et al, J. Phys. D: Appl. Phys., 36, (2003) 79.

[18] The author wishes to thank M. Salomé, D. Eichert, R. Tucoulou, S. bohic, G. Criado-Martinez, P. Bleuet, R. Baker and S. Labouré for their involvement in the development of the ID21/ID22 Xray microscopes. 